\title{
Corela
}

Cognition, représentation, langage

5-2 | 2007

Vol. $5, \mathrm{n}^{\circ} 2$

\section{Polysémie, prototype et invariant : le cas du verbe «manger » en dagara}

Pénou-Achille Somé

\section{OpenEdition}

\section{Journals}

Édition électronique

URL : http://journals.openedition.org/corela/368

DOI : $10.4000 /$ corela.368

ISSN : $1638-573 \mathrm{X}$

\section{Éditeur}

Cercle linguistique du Centre et de l'Ouest - CerLICO

\section{Référence électronique}

Pénou-Achille Somé, "Polysémie, prototype et invariant : le cas du verbe «manger » en dagara », Corela [En ligne], 5-2 | 2007, mis en ligne le 30 décembre 2007, consulté le 30 avril 2019. URL : http:// journals.openedition.org/corela/368; DOI : 10.4000/corela.368

Ce document a été généré automatiquement le 30 avril 2019

\section{(c) (i) (2)(2)}

Corela - cognition, représentation, langage est mis à disposition selon les termes de la licence Creative Commons Attribution - Pas d'Utilisation Commerciale - Partage dans les Mêmes Conditions 4.0 International. 


\title{
Polysémie, prototype et invariant : le cas du verbe " manger » en dagara
}

\author{
Pénou-Achille Somé
}

1 Les études antérieures (Delplanque 1986, Somé 1982 et 1992) ont montré que le dagara ${ }^{1}$, langue voltaïque parlée au Burkina Faso, fait partie de ces langues dont le stock lexical est relativement faible en extension, mais extrêmement riche en compréhension, au sens où la plupart des mots connaissent une quantité extraordinaire d'usages «figurés ». J'ai choisi de développer ici le cas du verbe di qui mérite particulièrement d'être porté à l'attention des sémanticiens non habitués à cette langue. Ce verbe peut en effet se traduire, selon le contexte, par " manger ", d'où l'idée de " dépenser, profiter, brûler, être usé, faire souffrir ... », mais aussi, de manière plus surprenante, par «s'appeler » et de là «avoir les mêmes propriétés que $\mathrm{x}$, atteindre l'excellence, atteindre son but, normaliser une situation", ou au contraire "ne ressembler à x que de nom", etc. Chacune des acceptions de di en dagara possède un terme proche (croquer, flamber, se lustrer, faire mal... être ou avoir, appeler, ressembler, réussir, réparer, etc.). Je me propose d'analyser ces acceptions tour à tour en montrant que di exprime toujours une valeur marquée par rapport à ses faux-synonymes.

2 Après une présentation sommaire du système verbal en dagara $(\$ 1)$, les paragraphes 2 et 3 exposeront les différentes acceptions de di en tant que verbe de processus et les paragraphes 4 et 5 les usages de di comme verbe d'état. A ce stade de l'exposé, ces acceptions seront classées de manière traditionnelle, en termes de métaphores et de métonymies. Cette vision des choses sera cependant discutée en $\$ 6$ en confrontant l'approche cognitiviste et l'approche constructiviste de la sémantique. Enfin, en \$7, je tenterai de dégager « l'invariant » qui permet d'embrasser la diversité des usages de ce verbe polysémique. 


\section{1- Propriétés grammaticales du verbe en dagara}

3

En dagara, un constituant verbal peut se présenter sous deux aspects, l'un non-marqué (aspect zéro), l'autre marqué (- $\mathrm{dv}^{2}$ : aspect inaccompli). La forme marquée caractérise un processus inaccompli (progressif ou habituel). La forme non-marquée, en elle-même, ne permet pas de concevoir le procès dans la durée: elle n'exprime qu'un événement ponctuel dans un récit ou dans l'injonction. Par ailleurs, ce constituant verbal peut être affecté ou non de l'actualisateur -na qui marque l'ancrage ou non dans la situation d'énonciation. Ce n'est que dans ce cas que l'aspect zéro prend la valeur d'accompli, ou plus exactement de parfait : «il a mangé ». Et la particule -na interviendra de manière significative dans l'une des acceptions de di.

(1) ...v di... $\mid . .$. v di-de... $\mid$ v di-ná $\mid$ v di-de-ná

... il manger | Il manger-inacc | Il manger-act | Il manger-inacc-act

...il mangea... | ...il mangeait... | il a mangé | il mange

ailleurs, la conjugaison verbale en dagara ne connaît pas de flexions de voix. La diathèse n'est donc marquée que par la syntaxe de l'énoncé : on a la possibilité de prendre le patient comme sujet, à condition d'évacuer l'agent. Toutefois, ces propriétés générales du verbe en dagara permettent de distinguer des classes de processus. Avec les verbes d'activité pure, le patient ne peut être sujet que si le verbe est à l'aspect inaccompli et avec le sens d'une voix moyenne :

Sujet agent Sujet patient

(2) ba pób-na a bibiid |* a bibiit ba pób-na ${ }^{3}$

Ils frapper-act les enfants $\left.\right|^{*}$ les enfants frapper-act

Ils ont frappé les enfants. |* Les enfants ont été frappés.

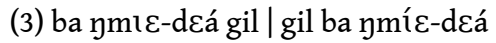

Ils jouer-inacc-act balafon | balafon jouer-inacc-act

Ils jouent du balafon | Le balafon, ça se joue.

(On ne fait pas n'importe quoi avec lui.)

5 Tandis qu'avec les verbes d'accomplissement ${ }^{4}$, on a, en plus de la valeur moyenne à l'inaccompli, une valeur stative à l'aspect zéro :

(4) v kaa-da-ná a Pùlé | a Pùlé kaa-da-ná

il casser-inacc-act la branche | la branche casser-inacc-act

Il casse la branche. | La branche se casse (ou est en train d'être cassée).

(5) v ka-ná a Pùlé | a Pùlé ka-ná

il casser-act la branche | La branche casser-act

Il a cassé la branche. / La branche est cassée.

En dehors des processus, le dagara possède un certain nombre de verbes d'état qui sont incompatibles avec l'opposition aspectuelle accompli / inaccompli. La plupart des états sont invariablement à l'aspect-zéro, comme "caa qu'à l'aspect marqué, comme "mii-de », être acide. On peut classer les acceptions de di selon que ce verbe traduit un processus ou un état.

\section{2- Processus « di » avec un sujet animé}

7 On verra que le sujet n'est pas forcément agentif et que, quand il est agentif, son rôle est complexe. Mais, dans tous les cas, son caractère 㦴 animé joue un rôle crucial. 


\section{1 - Avec un objet inanimé : « manger »}

8 Quand il réfère à un processus, le verbe di appartient à la catégorie des accomplissements. Pour le démontrer, je vais comparer $d i$ au verbe «/w每 $\mathrm{b}$ » qui lui ressemble à certains égards, tout en se distinguant systématiquement de lui, puisqu'il s'agit d'un verbe d'activité. D'abord, ils n'ont pas exactement le même comportement visà-vis de la diathèse, quand le sujet syntaxique n'est pas l'agent. Avec di, à l'aspect zéro, on obtient alors une valeur stative. Avec " $/ \mathrm{w}_{\text {Fog }}^{\mathrm{F}} \mathrm{b}$ ", cette tournure se rencontre plus volontiers avec une quantification de l'objet et traduit un processus accompli :

| * a sínbie - Pwób-ø-ã

(6) a saáb dí-ø-ná | a sínbie há Pwób-ø-á

Le to di-ACC-ACT | les arachides toutes croquer-ACT-ACC

Le to est mangés | Toutes les arachides ont été croquées

9 Cette différence n'est que l'une des manifestations des propriétés sémio-syntaxiques générales de nos deux verbes. Ils diffèrent quant à la nature du complément. Les locuteurs dagara ${ }^{6}$ définissent le verbe di par : « mettre objet en bouche pour l'avaler ». Et

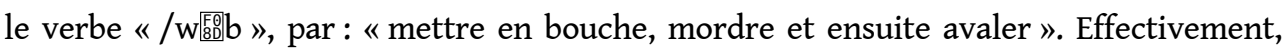
avec di, l'objet mangé est directement absorbable, l'absorption est inhérente au concept. Avec «/ $\mathrm{w}$ 渵 $\mathrm{b}$ » l'aliment n'est pas directement assimilable, son absorption demande un effort et n'est d'ailleurs pas garantie, car on peut dire :

(7) a u Pwob-ø-a tí bá vol $\varepsilon$

il croquer-ACC-ACT et NEG avaler NEG

Il a croqué, mais il n'a pas avalé;

mais pas :

(7) $b *$ * di-ø-ná tí ba vol $\varepsilon$

Il manger-ACC-ACT et NEG avaler NEG

* Il a mangé et n'a pas avalé.

Le complément de di est donc obligatoirement une matière pâteuse (le tô, pâte de haricot...). Au contraire, lorsque la matière absorbée est solide comme par exemple les arachides, le maïs, la viande, les ignames, les patates, le riz ${ }^{7}$, etc., c'est le verbe «/w嚂 $\mathrm{b}$ " qui est préféré au verbe di. Ainsi on dira :

(8) a bíe did-de-ná saáb

le enfant manger-INAC-ACT pâte de mil

L'enfant est en train de manger du tô.

(9) tì Pwob-do-ná sínbie

Nous croquer- ACC-ACT arachides

Nous sommes en train de manger des arachides.

11 Cette propriété occasionne une contrainte sur la sélection du sujet de di. En règle générale, on peut dire que, quand le mangeur est un animal, on n'utilise pas le verbe di. Pour les animaux domestiques comme le chat, les vaches, les chèvres, ou pour les animaux sauvages comme les hyènes, le lion, etc., le verbe «manger » se traduit par " / w医b ", qu'il s'agisse de l'herbe ou de la viande, et «ny媐@ $\mathrm{t}$ ” pour les petits animaux comme les agneaux, les cabris, etc.; dans l'un et l'autre cas le verbe prend obligatoirement un complément d'objet :

(10) nááb Pwób-do-ná muon

la vache croquer-INAC-ACT herbe

La vache broute l'herbe 
12 De même pour dire : la hyène est en train de manger une chèvre, l'agneau est en train de manger de l'herbe, etc. Quelques animaux font exception cependant, soit parce qu'ils sont incapables de croquer (poules), soit parce qu'ils sont capables de manger sans croquer (chien) :

(11) a a nuvt dìd-de-na kí les poules manger-INAC-ACT mil Les poules picorent le mil.

(11) b a báa dí-de-ná saáb

Le chien manger-INAC-ACT pâte de mil

Le chien mange du tô.

Il va de soi que ces contraintes sont levées quand l'animal est personnifié, notamment dans les contes (l'araignée, l'hyène, le lièvre, le lion, etc.), c'est le verbe di qui est alors utilisé.

Finalement, ces deux verbes mettent en œuvre deux types différents de transitivité, car

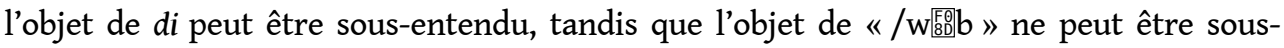
entendu que s'il peut être inféré dans le contexte :

(12) a à bibiit dí-de-ná ${ }^{-}$?wób-do-ná

les enfants manger-INAC-ACT

Les enfants sont en train de manger.

(12) b v dé-ø-n á sínbie Pwob

il prendre-ACC-ACT les arachides croquer

Il a pris les arachides et les a croquées.

15 Ceci veut dire que l'objet de di va de soi. De plus, la nourriture générique est désignée par

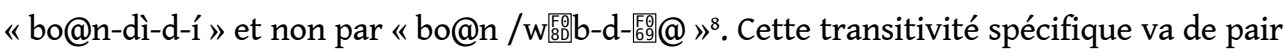
avec un sémantisme spécifique et plus précisément une différence de finalité. Quand il est intransitif, le verbe di réfère à un acte socialisé (il a mangé = il a pris son repas) et vital (il est rassasié). Le nom verbal « di-b » désigne le moyen de subsistance et par métonymie la vie. Voici un exemple qui apparaît dans le discours du griot à propos du chasseur :

(13) a ba tam mígd Pı-ø-ná bá dí-b

Le leur arc corde faire-ACC-ACT leur fait de manger

La corde de leur arc constitue leur moyen de subsistance.

Alors que «/w茴 $b$ » n'exprime qu'un acte de consommation, voire d'épuisement, dont il est possible de critiquer l'inutilité. Il s'agit d'un verbe d'activité pure, et non d'un accomplissement :

(14) a fúu yand fùun da líbie n-á fú Pwob

le pagne ce toi-que acheter argent c'est tu croquer

Le pagne que tu as acheté c'est de l'argent que tu as croqué (c'est de l'argent que tu as dépensé bêtement).

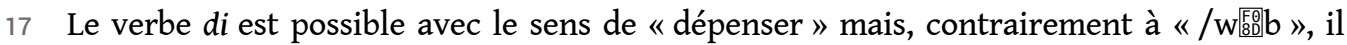
marque que la dépense est utile au sujet :

(15) a $v$ did-de-ná libíe à níbe Pyáng

il di-INAC-ACT argent les personnes pour

Il dépense de l'argent au service des gens.

(15) b a dába ba dì-ø a libí é

le homme NEG di-AOR le argent NEG

L'homme n'a pas dépensé l'argent (il ne l'a pas utilisé).

18 Une dernière précision à apporter est que, lorsque l'on absorbe la pâte de mil sans l'accompagner de sauce, on utilise le verbe "ga » à la place du verbe di qui, lui, suppose toutes les conditions favorables à l'absorption : 
(16) a bíe gáa-da-ná sáab

l'enfant manger-INAC-ACT pâte de mil

L'enfant est en train de manger la pâte de mil sans sauce.

19 En revanche, la sauce n'est jamais « bue », mais elle est "mangée », car dans la pratique des Dagara, elle accompagne normalement le plat de base, le tô. L'énoncé (17) peut être une simple invitation à manger le tô, par métonymie. Mais il peut aussi avoir son sens littéral, malheureusement. En effet, durant la période de soudure (juin-août), beaucoup de familles se nourrissent exclusivement de la sauce sans la pâte de mil du fait de la rupture des vivres (mil, maïs, etc.) :

(17) nyı wa dizı̀єd

vous venir manger sauce

Venez manger la sauce.

Cette finalité explique un premier type d'usage figuré, où la métaphore porte, non sur le verbe di lui-même, car il signifie bel et bien « manger », mais sur l'énoncé complet : c'est l'acte de consommation qui prend une valeur symbolique ou mythique dans la société dagara, en référant aux responsabilités parentales vis-à-vis de leurs enfants ou réciproquement. En donnant aux parents les parties du gibier tué désignées ci-dessous, l'enfant se met en conformité avec la règle sociale, et les parents reconnaissent l'appartenance de ce dernier à la grande famille ancestrale. Quand le fils a tué un gibier, la croupe revient à sa mère, les pattes reviennent à son père :

(18) a saan díd-de-ná bóngbec

le père manger-INAC-ACT pattes

Le père mange les pattes

(c'est au père qu'appartiennent les pattes du gibier tué par le fils)

21 Lorsque l'on tue une vache à la maison, c'est à l'aîné de la famille que reviennent les bouts de pattes de l'animal. Les responsabilités dans ce contexte sont les mêmes que celui du père qui reçoit les pattes du gibier tué par le fils : l'aîné est responsable de la gestion de la maison et donc de tous ceux qui y habitent.

Ceci montre qu'en dagara, le verbe di ne veut pas dire simplement "absorber » car cet acte est toujours orienté en fonction d'une norme, non seulement physiologique ou culinaire, mais également sociale et cet acte s'inscrit dans un espace centré sur l'Homme. Cette valorisation inhérente à di éclaire la plupart de ses usages figurés.

\section{2 - Avec un objet animé : emplois métonymiques}

Si di est suivi d'un complément animé, celui-ci renvoie implicitement aux propriétés de ce complément. Ce genre d'énoncé met en avant les responsabilités sociales :

(19) v di-de-ná a vì saan

il/elle manger-INACC-ACT le son père

Il absorbe (les biens qui lui sont procurés par ) son père

On pourrait dire de la même façon : il absorbe (les biens qui lui sont procurés par ) son fils; l'enfant bénéficie de (les acquis matériels procurés par) son oncle maternel, etc. Précisons cependant que cette métonymie est possible, même quand il n'existe pas de lien de parenté entre le sujet et l'objet :

(20) tı did-de-ná Penv a daa puว

nous di-INACC-ACT Pénou le marché dans

Nous jouissons de la générosité de Pénou au marché. 

tâche du fossoyeur. Celle-ci occasionne un autre type de repas organisé à la suite d'un enterrement et dont le but est de prévenir tout danger pouvant émaner de l'âme de celui que l'on vient d'enterrer.

(22) c tı dí-de-na bo

nous manger-ACC-ACT le (rituel) du trou

Nous sommes en train de manger le trou (nous mangeons le repas organisé après

l'enterrement). 


\section{4 - Avec un objet animé : emplois métaphoriques}

30 Lorsque l'objet est un humain non-responsable, la métonymie se combine à la métaphore, car alors il n'est plus question de manger, contrairement à ce qu'on a vu en 2.1, 2.2 et 2.3. Les exemples de ce type montrent que di peut impliquer aussi bien une charge qu'un profit et nous introduisent à la conception de la vie des Dagara, conception qui admet un échange d'enfants entre un fils aîné et son frère cadet.

(23) v di-de-ná à v kpécn bíe

il manger-INACC-ACT le son grand frère fils

Il a hérité de l'enfant de son frère aîné (selon la coutume).

Il ne s'agit pas d'une « adoption d'enfant » au sens occidental du terme, à savoir lorsqu'il y a, d'une façon ou d'une autre, une rupture avec les parents biologiques. Leverbe di est réservé au cas particulier de l'adoption d'un enfant du frère décédé ${ }^{10}$. En pareille circonstance, chez les Dagara, chacun est amené à traiter les orphelins de son frère exactement comme ses propres enfants. L'énoncé (23) prend ainsi le sens de : il mange à vie les « attributs » de l'enfant, et en conséquence, il devient socialement responsable de lui à l'instar de son propre enfant: il doit l'éduquer, l'initier aux choses de la vie, le marier, etc. ; en retour l'enfant adopté assume toutes les responsabilités d'un fils vis-à-vis de son père.

\section{5 - Avec un objet inanimé : emplois métaphoriques}

Dans les exemples du type "pardon", on a un usage métaphorique du verbe di car on n'absorbe aucun plat. De plus, on a un usage métonymique de l'objet, le cœur désignant ce qu'on a sur le cœur. Pardonner, c'est résorber ce qu'on a sur le cœur. Cela dit, la finalité du pardon est comparable à celle des exemples précédents, car cette notion contient bel et bien l'idée de normalisation ou d'annulation du mal, comme on l'a vu pour « manger le meurtre ou le trou ».

(24) a dába dí-de-ná súgd kud-do

l'homme manger-ACC-ACT cœur donner-INAC lui

L'homme est en train de lui pardonner.

Les emplois métaphoriques 2.4 et 2.5 introduisent donc une variable importante car ils montrent que, même en l'absence d'absorption alimentaire, di marque toujours le centrage de l'activité humaine autour d'une norme. Les paragraphes 2.3, 2.4 et 2.5 ont en outre ceci de particulier que di marque une normalisation, c'est-à-dire la réparation ou la compensation d'une situation condamnable ou malheureuse.

\section{6 - Avec objet abstrait : emplois métaphoriques}

Le complément peut être un nom abstrait exprimant un état, souvent dérivé d'un nom concret, contrairement à celui de 2.3 qui exprimait une action. Dans ce cas, il ne s'agit plus de "manger", mais l'objet exprime un jugement de lénonciateur sur le comportement du sujet. Voyons d'abord en quoi cette qualification se distingue des

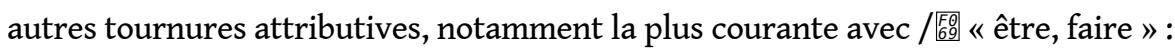

(25) a v did-de-ná gàndaa-lví:

il di- INAC-ACT bravoure

Il « mange » la bravoure (il est vraiment brave). 
(25) b v Pıd-d $\varepsilon$-na gàndaa-lv

il faire-INAC-ACT bravoure

Il pose des actes de bravoure.

Il faut d'abord noter que le verbe /葛 a une distribution très large et un sémantisme faible.

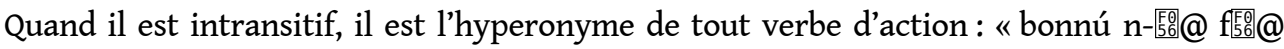

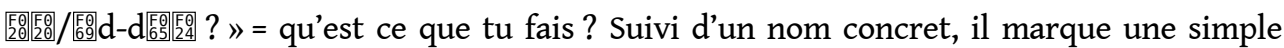
identification (l'enfant est un garçon, l'objet est en bois). Suivi d'un adjectif, il traduit l'état du sujet (l'animal est trapu, cet homme est mauvais) à l'aspect zéro ou son devenir à l'aspect inaccompli. Alors que di ne peut pas être suivi d'un adjectif :

(26) a a kpadú Pı-ná zì a kpadu Pıd-dè-ná zì

l'habit être-ACT rouge l'habit être-INAC-ACT rouge

L'habit est rouge. L'habit devient rouge.

(26) b * a kpadú di-ná zì * a kpadú dìd-de-ná zì $\varepsilon$

* l'habit di-ACT rouge * l'habit di- INAC-ACT rouge

En fait, conformément à sa nature (cf. 2.1), di n'est ici compatible qu'avec les abstractions qui impliquent un accomplissement. D'une part, ce qui est évalué avec $d i$, ce n'est pas tant le sujet lui-même que son activité. Celle-ci est un procès implicite, mais qui apparaît dans le contexte ; par exemple, il mérite l'appellation de «brave » pour avoir osé partir à la chasse sans fusil ou avoir affronté le lion et l'avoir tué avec un simple gourdin. D'autre part, et surtout, il exprime, non une simple qualité, mais l'excellence dans cette qualité : il a atteint le plus haut degré, il a fait tout ce qui est nécessaire pour mériter le titre de «brave ». Autre exemple :

(26) c a dába dí-de-ná náalvo

l'homme di- ACC-ACT richesse.

"Cet homme mange la richesse » = il mérite d'être appelé « riche»

par exemple, pour être capable de donner tant de cadeaux ou de dépanner financièrement tous les nécessiteux qui se présentent à sa porte. Dans certains cas, l'opposition «/熈 》 versus di correspond clairement à celle qui existe entre la qualité pure et l'occurrence contingente :

(27) a fu did-de-ná $n$ dánstó $\varepsilon$

tu di- INAC-ACT mon témoignage

Tu es mon témoin = tu assumes pleinement le témoignage en ma faveur.

8 Avec ce genre de notion, si on utilise le verbe / / $\left[{ }_{09}\right.$ 《 faire » à la place de di, il faut alors

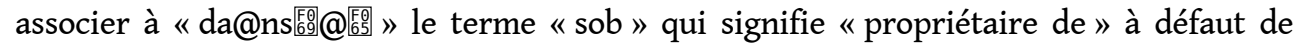
quoi, l'énoncé n'est pas valable :

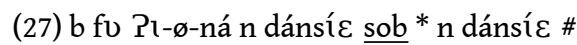

toi faire-ACC-ACT mon témoignage-propriétaire

Tu me sers de témoin

Dans ces exemples, le verbe di nous introduit à l'intérieur du domaine notionnel, tandis

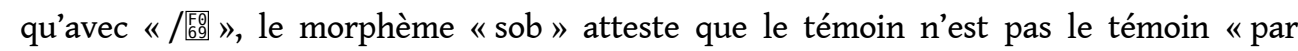
excellence ", mais qu'il est seulement utilisé comme simple occurrence de témoin, un témoin quelconque. Corollairement, le jugement exprimé par di a pour effet de surpasser tous les concurrents possibles; cet effet particulier est obtenu lorsque di est suivi du nom concret correspondant au dérivé abstrait :

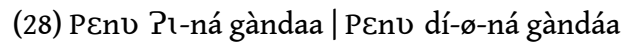

Penou être-act brave | Penou di-ACC-ACT brave

Penou est un brave. | Penou est le brave des braves. 
ncé de gauche traduit une simple attribution; alors que l'énoncé de droite avec di présente le sujet comme cas unique, ce qui, d'ailleurs, peut être perçu comme une forme de provocation aux yeux des autres.

41 Par ailleurs, le statut qualificatif de l'objet abstrait a une conséquence importante sur la valeur axiologique de l'énoncé. Dans les paragraphes précédents, en effet, j'ai montré que di impliquait toujours une valorisation de la part de l'énonciateur: l'objet étant considéré comme bon, favorable pour le sujet et approuvé par la Société. Or, ici, la valeur axiologique est inhérente à l'objet et peut, de ce fait, être positive ou négative, favorable ou défavorable, laudative ou péjorative. Soient les exemples suivants :

(29) a a dába dí-de-ná suon

l'homme di- INAC-ACT sorcellerie

L'homme est en train de manger la sorcellerie (l'homme est en train de poser des actes propres aux sorciers).

par exemple : il faut être sorcier pour pénétrer dans cette forêt dangereuse en pleine nuit, ou pour grimper à cet arbre de dix mètres sans l'usage d'une corde. De même, on dirait que quelqu'un est en train de manger le drame (yéng@) s'il continue à se piquer avec des flèches empoisonnées ou s'il se permet de boire du pesticide. On pourrait multiplier les exemples avec le complément "folu», l'inconvenance : l'homme se comporte comme un "sagouin " puisqu'il se permet de détourner la femme de son frère ou de son cousin, ou s'il refuse de fournir la dot sa belle-fille parce qu'il a dilapidé tous les biens de la famille. Ou

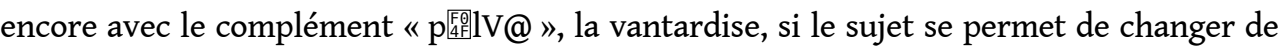
tenues vestimentaires deux fois par jour, ou s'il se permet d'attirer l'attention des gens sur son habillement. L'exemple suivant est doublement métaphorique, puisque « manger la saleté » veut dire "effectuer une tâche repoussante». En l'occurrence, il s'agit de déterrer un mort, dont on sait qu'il est en décomposition.

(29) b a dába dí-de-ná dếg

l'homme di-INAC-ACT saleté

Le fossoyeur est en train d'effectuer un sale boulot.

Voici une brève explication : dans le mois de mai, en 2004, on a enterré un homme, dans un village au sud-ouest du Burkina Faso, avec ses vêtements comme il est usuel de le faire. Or une sécheresse qui a sévi dans cette période a été interprétée par les prêtres traditionnels comme étant liée au mort enterré un mois plutôt. Selon eux, cet homme dont le grand-père appartient à la grande famille qui se réclame comme descendant du dieu de la pluie - ne devrait emporter avec lui aucun tissu dans la tombe. On a donc ordonné séance tenante que le corps soit déterré et les habits ôtés avant de procéder à un

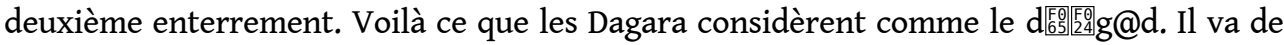
soi que cette situation a exigé ensuite que le fossoyeur "mange le trou», au sens réparateur que nous avons décrit au $\S(2.3)$.

Certains noms d'état sont ambigus et peuvent être valorisés différemment selon le

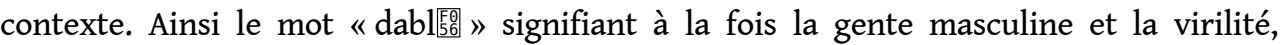
l'énoncé suivant pourra être interprété comme élogieux à l'aspect zéro :

(30) Pعnv dí-ø-na dàblú

Penv di-ACC-ACT virilité

«PEnv a mangé la virilité » = il a vraiment mérité d'être appelé « homme ».

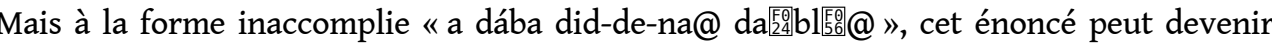
péjoratif, le sujet étant jugé comme quelqu'un qui s'affiche pour faire montre d'une force virile et brutale. 

《 n le mot túo « souffrance », le contraire de $n$ 瀶區 $n$. Dans ce contexte, les Dagara préfèrent le verbe won, qui signifie en fait « entendre ${ }^{11}$ » et est glosé ci-dessous par « ressentir » :

(31) a a dába dí-de-ná nvìion

l'homme di-INAC-ACT ce qui est bon

L'homme éprouve un vif plaisir.

(31) b v won-ø-ń túo * v di-ø-ń tuo

il entendre-ACC-ACT souffrance

Il a souffert (il a ressenti la souffrance).

On peut dire aussi «won n venant de l'extérieur. Alors qu'avec «di n qu'il fait volontairement. On peut donc penser que "won túo» c'est ressentir une souffrance qui a été imposée de dehors (le mauvais sort, la nature, les esprits, etc.) à l'individu et que pour cette raison la langue rejette « di túo » qui laisserait croire que non seulement l'individu accepte volontiers sa souffrance mais qu'il a pris en plus sur lui le désir de vivre cette souffrance, ce qui serait incompréhensible. Cela ne signifie pas que l'expression « di túo » n'existe pas en dagara. C'est en fait une expression qui est aussi disponible dans la langue mais avec une signification totalement différente :

(31) c tá wà di-túo wáa-dà ká $\varepsilon^{\prime-\zeta}$

NEG modalité di + souffrance venir-INAC ici NEG

Ne te donne pas la peine de venir ici.

Cette expression est toujours associée à une négation et prend le sens de «se donner la peine de, se fatiguer pour réaliser telle ou telle action » : le verbe di exprime donc une activité volontaire pour atteindre un but. Par conséquent, di est caractérisé par deux effets contradictoires : (i) le sujet est récepteur de l'objet, soit parce qu'il l'absorbe, soir parce que l'énonciateur lui attribue une propriété : (ii) le sujet est actif et il est la cause d'un changement d'état. Mais on s'apercevra que le second effet est contingent.

\section{3- Processus « di » avec un sujet inanimé}

Si le sujet est inanimé, on doit s'attendre à une modification de la visée, dans la mesure où une chose n'est pas dotée de volonté: d'une manière générale, lorsque di a un sujet inanimé, l'énoncé prend un sens détérioratif. De plus, s'il s'agit d'un processus, l'objet est lui aussi inanimé. L'effet métaphorique est évident.

\section{1 - Destruction totale}

Dans le premier cas de figure, le sujet typique est le «feu », notion inanimée mais très marquée dans la culture dagara comme une puissance (quasi agentive) de connotation négative. Ainsi quand on dit de quelqu'un que :

(32) v be-ń vùun pu

Il exister-ACT feu dans

Il est dans le feu, il a le feu aux trousses.

51 cela veut dire que l'intéressé est vraiment dans de sérieuses difficultés. Dans les exemples ci-dessous, vuun est le sujet et l'objet est tout ce qui peut être consumé par lui (l'habit, le bois, le champ, le mil, etc.) : 
(33) a a vuun dí-de-ná // dí-ø-ná a kpàdvo-

le feu di-INA-ACT // di-ACC-ACT l'habit

Le feu est en train de brûler l'habit // a brûlé l'habit semantiques particuliers :

(33) b * a vuun dí-ø-ná a vuun díd-de-ná

* le feu di-ACC-ACT le feu di- INAC-ACT

L'énoncé à l'inaccompli peut signifier que le feu sur lequel l'aliment est posé (la sauce, le riz, la soupe, etc.) est en train de s'activer. Il peut aussi marquer une forme de datation propre à la tradition orale, en faisant référence à la fin des feux de brousse située généralement vers fin mars. Ainsi, l'exemple ci-dessous signifie que quelqu'un est mort dans le courant du mois de mars.

(33) c a vuun díd-de-ná tı v kpí-ø

le feu di- INAC-ACT et il mourir-AOR

Le feu de brousse était en train de brûler quand il est mort

\section{2 - destruction potentielle}

54 On peut rattacher à ces acceptions les cas où di est utilisé par extension en parlant des outils tranchants comme le couteau, la hache, etc., pour lesquels on emploiera des expressions comme s[50

(34) a suo díd-de-ná * dí-ø-ná

Le couteau di- INAC-ACT * acc

Le couteau est tranchant

Mais je souligne que ces expressions traduisent une qualité, un processus virtuel et non un processus accompli ou en cours d'accomplissement. De plus, on réfère alors à l'état optimal d'un outil qui, pour être utile, ne peut être que dangereux.

\section{3 - destruction partielle}

En dehors du "feu», di ne peut exprimer un processus détérioratif avec un agent explicite. La cause externe peut être la marche, la route, les intempéries, etc. Et l'énoncé traduit l'usure, c'est-à-dire - je le souligne - un processus qui n'aboutit pas à l'anéantissement de l'objet, contrairement au feu :

(35) a a u negsó gbę dí-de-ná // dí-ø-ná

le son vélo pieds di- INAC-ACT // di- ACC-ACT

Les pneus de son vélo sont en train de s'user // sont usés.

(35) b* a sod did-de-na negsó gbę

* la route di-INAC-ACT vélo pieds

La route use les pneus.

On aurait la même chose avec « son fond de culotte est usé " //* la chaise a usé son fond de culotte. Ou : « les baguettes du balafon s'usent » //* les lames du balafon sont en train d'user les baguettes.

\section{4 - la relation sujet-objet}

La différence de transitivité entre 3.1 et 3.3 peut s'expliquer par le fait que l'usure n'est qu'une destruction partielle : l'objet existe encore, même s'il est mis hors d'usage. Alors 
qu'avec le feu, di conserve pleinement son statut d'accomplissement : détruire jusqu'au bout.

On peut aussi interpréter l'usure comme un processus dont l'agent et le patient coïncident (processus autonome comme dans «la branche casse»). Dans cette perspective, la route, les lames du balafon ne seraient pas tant les causes que les lieux de l'usure. Alors que le feu est un phénomène complexe : d'une part, il est une puissance, donc un véritable agent, même s'il n'est pas doué de volonté ; mais d'autre part, si le dagara peut concevoir le feu en lui-même, il sait aussi que le feu a besoin de consumer

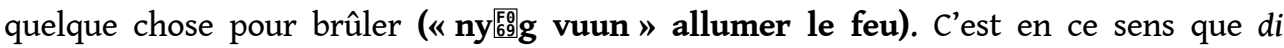
intervient pour appréhender un phénomène intrinsèquement relationnel qu'on trouve avec une minorité de verbes dagara: rencontrer, prêter (emprunter), têter (allaiter). Effectivement, quand di veut dire "brûler ", les deux termes $\mathrm{S}$ et $\mathrm{O}$ ont le même statut [concret] \& [-animé], ce qui permet la réversibilité syntaxique de la relation, même si le statut agent-patient reste constant :

(36) a a kpadv - dì-de-ná // dì-ø-ná vùun

l'habit di- INAC-ACT // di-ACC-ACT feu

L'habit est en train de prendre feu // a pris feu.

(36) $b$ a púo dì-de-ná // dì-ø-ná vùun

le champ di- INAC-ACT // di-ACC-ACT feu

Le champ est en train de prendre feu // a pris feu

Cette équivalence de statut s'observe également lorsque di est intransitif avec un sens analogue. Le sujet peut être le patient (l'habit, le bois, le champ, et) avec l'agent «feu » sous-entendu :

(36) c a kpadu did-de-na

le habit di-INAC-ACT

L'habit est en train de prendre feu (l'habit est en train de brûler)

\section{4- Etat « di » avec un sujet animé}

61 Examinons à présent les usages où di n'est pas compatible avec l'inaccompli. Le verbe di donne alors au prédicat une valeur stative, quasi-adjectivale. Le sujet alors n'est plus "agent ", mais possesseur ou attributaire d'une qualité ou d'une propriété. Toutefois, avec le verbe di, la visée demeure irrémédiablement mais elle est assurée par un agent extérieur qui est l'énonciateur.

\section{1 - L'objet est un nom propre}

Le nom individuel est la trace d'un événement passé où un père, une mère ou un ancien a attribué le nom (" pod » = nommer) à l'individu à sa naissance. Voici comment dire « $\mathrm{S}$ s'appelle 0 » en dagara :

(37) a a sáanna dí-ø-ná ká bangna (* did-de-na)

l'étranger di-ACC-ACT que Bangna * inacc

L'étranger s'appelle Bangna

63 Plus souvent, le sujet humain est déterminé par le mot « yúod» qui signifie « prénom ». En voici une illustration :

(37) b a u yúod dì-ø-ná ká Pعnv (* did-de-na)

le son prénom di-ACC-ACT que PEnv * inacc

Son nom est PEnv, il s'appelle PEnv 
a" est en fait la trace d'une énonciation première, car c'est ce connecteur qui permet d'introduire les propositions complétives ${ }^{12}$ : il dit que, il veut que, il demande si... Par exemple :

(38) dolúon yel-ø-ná ká a saa na wá-ø-ná

Donlúon dire-ACC-ACT que la pluie FUT venir-ACC-ACT

Donlúon a dit qu'il va pleuvoir

par le seul relateur « ka»:

(37) c a v yúod ka Penv

le son nom que Penv

Il s'appelle Penv

D'une certaine manière, ce connecteur fait de di un verbe d'énonciation. Mais contrairement à ce qui se passe dans le discours rapporté (37), ici l'énonciation première est occultée: la donation du nom ne se manifeste plus qu'à travers son résultat. Le caractère non-agentif du sujet apparaît lorsqu'on compare ce genre d'expression avec le

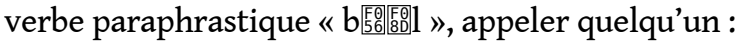

(39) tı buolo-ná à bíe Pland ka gòba

nous appeler-INAC-ACT l'enfant ce que goba

Nous appelons cet enfant "Goba » (le Gaucher).

67 Avec «b constructeur de la prédication. Tandis qu'avec di, le sujet est $\mathrm{C} 1$, récepteur d'une prédication pré-construite par un personnage Co extérieur à l'énoncé. De ce fait,

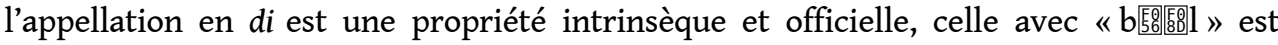
contingente, voire contestable : « Goba » est ici un surnom que nous donnons à l'enfant.

Il arrive cependant que le sujet coïncide avec le donneur du nom $(\mathrm{C} 1=\mathrm{C} 0)$, lorsque quelqu'un se donne volontairement un nouveau nom :

(40) a ba ha dína a ywée (* did-de-na)

ils tous di-act les prénoms

Ils se sont tous donnés des prénoms (ils ont pris des prénoms)/

(40) b v yó-ø-ná tı v bá dì yúod é

il voyager-ACC-ACT et il Neg. di- prénom Neg.

Il a voyagé mais il n'a pas changé de prénom.

On remarque dans ce cas, que le connecteur « ka » disparaît, de la même manière que dans les complétives dont le sujet est identique à celui de la principale :

(41) a * $n$ bó-da-na ká $n$ cend.

* Je vouloir-INAC-ACT que je partir

* Je veux que je parte.

(41) b n bo-íb-da-na céndú

Je vouloir-INAC-ACT le fait de partir

Je veux partir.

70

Le contexte du changement de nom (sans « ka ») peut se décrire comme suit: entre l'âge de 18 à 20 ans, au lendemain des indépendances, les paysans dagara se rendaient - le phénomène étant aujourd'hui fortement ralenti du fait des perturbations socio-politiques en Côte d'Ivoire - dans les plantations des pays côtiers (le Ghana, la Côte d'Ivoire) et, après un séjour de quelques années, ils revenaient au village en adoptant un nouveau "yu @od" ou prénom. Cette adoption de nouveau nom est vécue comme un rite de passage de la jeunesse à la vie adulte : n'avoir pas voyagé une fois dans sa jeunesse est une preuve 
d'incompétence, ce qui ne manque pas de provoquer les railleries des camarades de la même classe d'âge et même de la société.

Il ne faudrait pas confondre ce changement de prénom avec une autre situation que l'on peut gloser par : "acquérir de la popularité, de la réputation". Dans un tel cas, les Dagara abandonnent le verbe di au profit du verbe tEd "posséder", ou p䳸@g "acquérir, avoir". Ainsi pour dire que quelqu'un a acquis de la popularité, les Dagara profèrent les énoncés suivants :

(42) ted-ø-ná yúod | v póg-ø-ná yúod

il avoir-ACC-ACT nom $\mid$ il trouver-ACC-ACT nom

Il a une réputation (il est populaire). I Il a acquis une réputation.

La différence avec di (39a et 39b) est qu'ici le donneur de nom n'est pas le sujet, mais la communauté qui reconnaît la valeur du sujet et les services qu'il a rendus. De plus, le nom yuod prend le sens métaphorique de " réputation », celle-ci pouvant s'étendre au-delà de la communauté et des frontières du village : «a V yúod yí-O-ná » son nom est sorti (il est populaire, il a de la réputation).

\section{2 - Avec un nom abstrait}

73 Examinons à présent un cas de figure hybride. D'une part, la construction est ici normalement intransitive. D'autre part, le sujet est normalement un nom verbal, qui n'est pas formellement un sujet inanimé mais qui, par métonymie, renvoie à un agent animé. Et cette construction exprime la valorisation d'un accomplissement, sans que l'on se préoccupe du processus qui a permis ce résultat.

Un premier exemple est celui de "gba » qui veut dire «jouer pour de l'argent ». Le nom verbal "gba@ng» désigne un jeu qui consiste à lancer en l'air quatre cauris dont les positions gagnantes sont les suivantes : deux des cauris doivent être tournés vers le ciel et les deux autres tournés contre le sol; ou encore, il faut que les quatre cauris soient tous tournés vers le ciel ou tous tournés contre le sol. Lorsque l'on veut saluer un joli coup ou la victoire d'un joueur, on dit :

(43) a v gbáng dí-ø-ná* díd-de-ná

le son jeu di- ACC-ACT * inacc

Son jeu a gagné, il a fait un joli coup.

Avec ce verbe-là, l'agent animé ne peut être sujet : * 榴 di-na@ gba@ng (* il a gagné le jeu). Un autre cas intéressant est celui du verbe «/y医

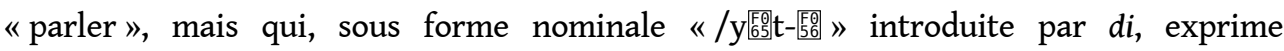
l'excellence de la parole et l'idée qu'un certain but est vraiment atteint. Comparer :

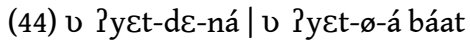

Il parler-INAC-ACT | Il parler-ACC-ACT finir

Il parle. | il a fini de parler.

(45) a a v Pyعtu dí-ø-ná * did-de-ná

la sa parole di-ACC-ACT * inacc

Il a prononcé le verdict, son jugement est sans appel.

Contrairement au cas précédent, on peut cette fois avoir l'agent humain en position sujet, justement parce que / $\mathrm{y}_{5.5}^{\mathrm{F}} \mathrm{t}$, contrairement à «jouer », est un verbe de parole et renvoie donc à une situation de locution. L'énoncé (44a) valorise plutôt le contenu du propos, tandis que dans (44b), c'est plutôt le sujet-orateur qui est valorisé : 
(45) b v di-ø-ná ?yetu

Il di-ACC-ACT parole

Il a bien parlé, il a raison.

Le jugement de valeur est prononcé à l'adresse d'un interlocuteur collectif: pour

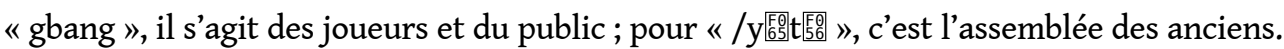
Et la scène se déroule en présence des spectateurs qui sont juges: il peut s'agir d'un

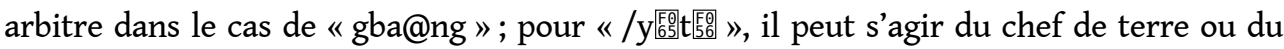
commissaire de police. Et le procès est caractérisé par un enjeu important : dans le cas de "gbang», c'est une somme d'argent; dans le cas de «/y熙t熙 », les propos de l'orateur doivent permettre de trancher une question diffcile et d'arriver à un verdict qui emportera l'adhésion de tous.

L'usage du verbe di dans ce contexte peut solliciter un complément d'objet. Le cas le plus

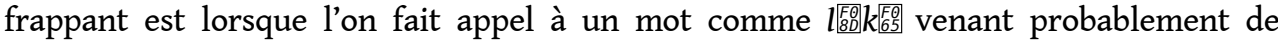
«luck» en anglais. Cet énoncé indique que l'orateur a réduit les objecteurs au silence, notamment dans le cadre d'une plaidoirie.

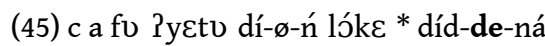

la ta parole di-ACC-ACT « luck »* inacc

Ta parole a mangé la chance (tu as obtenu gain de cause, face à la loi).

Il existe plusieurs autres possibilités de valoriser la parole avec d'autres verbes que di. Lorsque l'on est en dehors de toute situation conflictuelle et que le contexte nécessite cependant une appréciation parce qu'il s'agit tout simplement d'un arrangement amical ou d'une proposition destinée à fédérer plusieurs parties à une idée donnée, on utilise

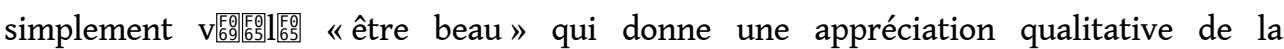
proposition qui n'a pas à être contrebalancée par une autre.

(46) a a fu Pyetu vícle-ná

la ta parole être belle-ACT

Tes propos sont beaux (tes propositions sont intéressantes).

Lorsque au contraire, on se trouve dans un contexte problématique ou polémique, on peut utiliser d'autres verbes que di. Il y a d'abord des verbes comme kp ${ }_{60}$ 《entrer » qui n'utilisent pas de complément mais qui prennent dend « celui de » comme sujet :

(46) b a fu dend kpé-ø-ná

Le toi propriété entrer-ACC-ACT

Ta propriété est rentrée (ce qui te revient est rentré) = tu as raison.

Il y a ensuite les autres verbes comme tu "suivre », dé " prendre », lo « tomber, prendre » ou encore lo di «tomber manger » qui utilisent un complément d'objet sód «route ", lequel prend aussi dans ce contexte la signification de « loi ». En voici des exemples :

(46) c a fu ?yctu tú-ø-ń sod

la ta parole suivre-ACC-ACT route

Tes propos suivent la bonne route ( $t u$ as raison).

Les énoncés ( $45 \mathrm{a}, \mathrm{b}, \mathrm{c})$ se distinguent de ceux en di en ce sens que l'énonciateur est conscient que cette appréciation de la situation n'engage que lui et lui seul. Alors que di exprime un accord évident pour toute la communauté : après tout, on n'est pas loin de la dénomination du paragraphe précédent (c'est ce qu'on appelle parler; voilà qui s'appelle jouer). 


\section{3 - Avec un objet animé : la ressemblance} le sujet et l'objet sont tous les deux des noms propres de personne ou lorsque le sujet est un pronom personnel et l'objet un nom de personne :

(47) a Zí́nnbe dí-na à dába yánd * dìd-de-ná

Zı̊nbe di-ACC-ACT l'homme cet * inacc

Zlenbe est le sosie de cet homme.

La traduction "être sosie de » est utilisée ici faute de mieux. Sur le plan sémantique, ce

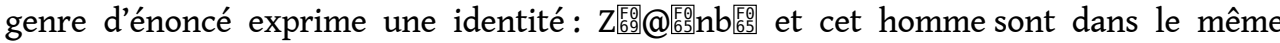
Intérieur, ils ne font qu'un. Cette construction avec di se distingue de la

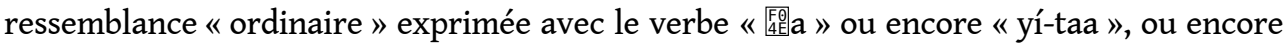
en recourant à un verbe quelconque combiné avec le morphème discontinu de comparaison ná....na :

(47) b fu ya-ø-ná Báaté

tu ressembler-ACC-ACT Báaté

Tu ressembles à Báaté

(47) c fuon ní à báaté yí-ø-ń taa // ná-ø-ń táa

toi et le Báaté sortir-ACC-ACT récipr // ressembler-ACC-ACT-récipr

Toi et Báaté entretenez une ressemblance :

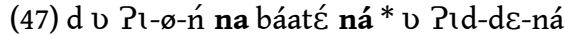

il faire-ACC-ACT comme Báaté comme

Il est comme Báaté (il est bâti physiquement comme Báaté).

La première différence est qu'avec les verbes « 殹a » ou « yí-táa » ou avec les connecteurs "na... na", le sujet est la source d'une impression 0 ressentie par un énonciateurrécepteur (il me fait l'effet de 0 ). Par contre avec le verbe di, le sujet est la cible du jugement $O$ émis par un énonciateur-constructeur (je le trouve 0$)^{13}$. La seconde différence est que di effectue le rapprochement $\mathrm{S}$ - $\mathrm{O}$ en éliminant toute différence possible entre les deux termes.

Dans l'exemple suivant, on a un sujet apparent qui est inanimé, mais qui, par métonymie, renvoie à un sujet humain (ton état $=$ toi). Le sujet peut représenter non une partie physique précise du corps, mais une «manière d'être » de la personne (l'odeur, le flux de la personne, les gestes, le regard, le sourire, etc.). Cet état peut être favorable, et dans ce cas, il se créera une entente très forte entre $\mathrm{S}$ et $\mathrm{O}$. Il peut être défavorable, et dans ce cas, l'énoncé marque une rupture entre eux, parfois sans qu'aucune raison apparente ne justifie une telle aversion. Les exemples suivants illustrent ce cas :

(48) a a u dunbo dí- $\varnothing-f u-n a *$ díd-de-fu-na

le son état di- ACC-toi-ACT * inacc

Sa manière d'être est en totale harmonie avec la tienne.

(48) $b$ a u dunbo ba dì fu $\varepsilon$

le son état neg di-acc toi Neg

Sa manière d'être n'est pas en harmonie avec la tienne (vous êtes tout le temps antagonistes).

Dans la ressemblance, on n'a pas « $S$ est $O$ », mais $S$ est traité comme $O$ par l'énonciateur. Cette identification exprimée par di a un intérêt particulier, car la signification de di ici est déterminée à la frontière de l'ethnologie et de la langue : employer di dans ce contexte suppose non seulement une maitrise de langue mais aussi une connaissance des règles sociales, à savoir qu'il est malséant de comparer ainsi quelqu'un à un membre de la 
famille proche : un père et son fils, sa fille, une mère et ses enfants. Ce tabou repose sur une croyance populaire selon laquelle une telle comparaison attire l'appétit des sorciers. Et pour empêcher un sorcier de nuire à l'enfant qui ressemble à son père, à sa mère, on lui met au poignet un bracelet de protection contre tout esprit maléfique.

Enfin, il paraît important de signaler une particularité énonciative propre à cette expression de la ressemblance. En fait, les énoncés comme (46a, 47a, 47b) marquent tels quels (affectés de l'actualisateur -na) une similitude indiscutable de $\mathrm{S}$ et 0 . Or, la manière la plus courante d'exprimer la ressemblance avec di est dépourvue de l'actualisateur na :

(49) a Donlúon dì-ø Báatć * dìd-de

Donlúon di-acc Báaté * inacc

Donlúon est le sosie de Báaté

(49) b Zí $b$ be dìd-ø à dába yánd * dìd-de

Zıenbe di-acc l'homme cet* inacc

Zıenbe est le sosie de cet homme

89 Je souligne que c'est la seule acception du verbe di qui autorise cette nuance. Faute de place, je ne peux que résumer ici la valeur de la présence / absence de cet actualisateur. Celui-ci est absent à chaque fois que le propos n'est pas ancré dans la situation de l'énonciateur (mode inactuel): à l'impératif, au mode narratif et dans certaines subordonnées, notamment l'hypothèse. Au contraire, -na indique que le propos est entièrement pris en charge dans l'espace-temps de l'énonciateur (mode actuel). Dans le cas de la ressemblance avec di, l'actualisateur -na marque une adhésion sans réserve de l'énonciateur à son énoncé : "S est identique à $\mathrm{O}$, c'est évident pour tout le monde. $\mathrm{Au}$ contraire, on peut dire que l'absence de -na marque une indétermination, ou une distanciation de l'énonciateur. " $\mathrm{S}$ est identique à $\mathrm{O}$, si je ne m'abuse, mais je peux me tromper".

Reste à expliquer pourquoi "s'appeler" exige -na, alors que "ressembler" ne l'exige pas. Dans "s'appeler", la dénomination est conforme à la donation du nom qui est préconstruite: le mode actuel est alors obligatoire. Alors que dans "ressembler", la propriété n'est pas pré-construite et l'identification est a priori approximative, voire abusive: on a vu justement que la ressemblance avec di ne peut être formulée qu'en tenant compte des règles de bienséance. Ce n'est que lorsque la ressemblance est évidente pour tout un chacun que l'actualisateur -na peut apparaître.

\section{5- Etat « di » avec un sujet inanimé}

91 Comme on l'a vu plus haut, l'incompatibilité avec l'inaccompli marque une absence d'agentivité et, en même temps, une forte présence de l'énonciateur. De plus, comme nous l'avons avec les acceptions processives, la présence d'un sujet inanimé donne à l'énoncé une valeur détériorative ou péjorative.

\section{1 - le sujet est une partie du corps : la souffrance}

92 Nous avons pu comparer plus haut les deux verbes di et / $w$ Fi⿺辶⿸ Ces deux verbes s'opposent également dans l'expression de la souffrance. Avec «/W医b ", verbe d'activité, la douleur est un processus, ressenti quand le verbe est à l'inaccompli, 
révolu quand le verbe est à l'accompli. Tandis que la douleur exprimée avec di est un état actuel, caractérisé par l'aspect zéro :

(50) a a pád ?wob-do-ma na $\mid$ a n tobd ?wob-ø-na

le anus faire mal-INAC-moi-ACT | l'oreille croque-acc-act

L'anus me fait mal. |J'ai eu mal à l'oreille.

(50) b a pád dí-ø-ma-na | a n tobd dí-ø-ma-na

le anus di-ACC-moi-ACT $\mid$ mon oreille di-acc-moi-act

J'ai des hémorroïdes. |J'ai l'otite.

Dans les deux cas, le sujet est une partie du corps et l'objet est un être animé qui ressent le mal. Mais avec di, le mal est diagnostiqué; on sait précisément qu'il est d'origine infectieuse ; le Dagara croit que c'est un mal grave qui peut entraîner la mort. Par contre avec «/W臨 $b$ » le mal est vague, on ne l'a pas diagnostiqué. Il peut s'agir d'un abcès ou simplement d'un coup reçu de l'extérieur.

La présence d'un sujet inanimé, avons-nous dit, engendre un effet détérioratif. Mais étant donné ce que nous avons affirmé à propos de l'enjeu du verbe $d i$, à savoir la vie, on peut dire que, cette fois, l'énonciateur ne fait pas simplement état d'une sensation douloureuse, mais va jusqu'à dire que sa vie est compromise, par le dysfonctionnement de son oreille, de son anus, etc., qui ne peuvent plus assurer leur fonction normale. D'ailleurs il est commun d'entendre les énoncés suivants :

(51) a n pád // a n tobd kúv-do-má-na

le mon anus // le mon oreille tuer-INAC-moi-ACT

le cul me tue (les hémorroïdes sont si avancées que je peux en mourir)

// mon oreille me tue (l'otite est si avancée que je risque d'en mourir).

Il faut par contre préciser que si la partie du corps qui fait mal est la n et que l'on utilise le verbe di il y a un changement de sens, car la langue impose alors la valeur « absorber » avec une référence quantitative :

(52) a a u nu-jod dì-ø-ná

la sa bouche di-ACC-ACT

Sa bouche a vraiment mangé (il a beaucoup mangé).

96 Cependant, si l'on veut bien marquer la présence d'un mal à cet endroit, telles que des plaies sur les gencives, on est obligé dans ce cas, de faire appel à un complément

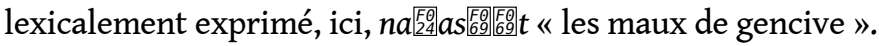

(52) b a v núod dì-ø-ná nàası t

la sa bouche di-ACC-ACT maux de gencive

Sa bouche a 'mangé' les maux de gencive = Il a des plaies sur sa gencive

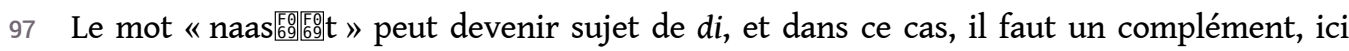
« $n$ [50 compatible avec l'inaccompli ; il s'agit donc d'un processus, comparable à « brûler » (\$.3)

(52) c v nàasıt dí-ø-ná ù núod

les maux de gencive di-ACC-ACT sa bouche

Les maux de gencive ont " mangé » sa bouche = il a des plaies sur sa gencive

(52) d nasut díd-de-ná à u núod

maux de gencive di-INAC-ACT la sa bouche

Des maux de gencive sont en train d'attaquer sa bouche

98 Le verbe «/ $\mathrm{W}$ 蔫 $\mathrm{b}$ 》 serait possible pour dire : la bouche me fait mal, mais impossible dans * les plaies blessent ma bouche.

Pour les autres parties du corps, la langue fait appel à des verbes autres que di. Ainsi lorsqu'il s'agit d'exprimer par exemples les maux d'yeux et de ventre, on utilise le verbe 


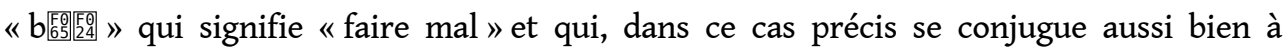
l'inaccompli qu'à l'accompli, ce qui fait de lui un verbe processif.

(53) a a n pús // nínbie bį̀de-má-na

le mon ventre // mes yeux faire mal-INAC- à moi-ACT

Mon ventre // Mes yeux me fait/font mal

(53) b a n púว // nínbie be-ø-má-na

le mon ventre // mes yeux faire mal-ACC-moi-ACT

Mon ventre // Mes yeux me fait / font mal

Le verbe zab "faire mal» qui est le même verbe que «faire la bagarre, exprimer verbalement une colère", peut s'employer aussi dans ce contexte aux deux aspects, à l'accompli et à l'inaccompli :

(53) c a n púo // nínbie zab-da-má-na // zá-ø-ma-ná

le mon ventre // mes yeux faire mal-INAC-moi-ACT // faire mal-ACC-moi-ACT

Mon ventre // Mes yeux me font mal // m'ont fait mal.

101 Pour le reste des parties du corps qui souffrent d'un mal quelconque, la tête, les dents, les jambes, le cou, la poitrine, la colonne vertébrale, le genou, les côtes, la main, les doigts, les pieds, les doigts du pied, le talon, les tempes, les joues, etc., la langue renvoie au seul verbe « / W區 $b$ » - et non à di - comme dans l'exemple (49a).

On peut se demander ce qui fait la spécificité des oreilles et de l'anus par rapport aux autres parties du corps. Peut-être s'agit-il des « orifices », auquel cas ces parties seraient conçues comme des passages par où un mal extérieur peut pénétrer dans le corps humain. Cette hypothèse est cependant difficile à vérifier. D’une part, le nez ne peut être " mangé »; mais il faut savoir que cet organe est associé au souffle (l'expression « ny

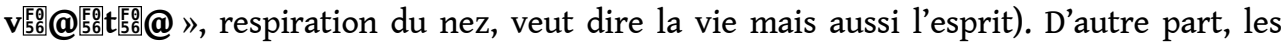
orifices correspondant aux organes génitaux sont des tabous dont on ne parle pas, surtout s'il s'agit d'infection.

\section{2 - le sujet est un nom abstrait : la pénurie}

103 Par ailleurs, di peut, par métaphore, référer à un manque, ou à toute sorte de phénomène qui met en jeu la survie de l'individu ou de la communauté. Le sujet est alors un nom abstrait de sens privatif comme náng "pauvreté », éventuellement avec un déterminant concret comme l'argent, l'eau, le mil, etc.

(54) a a náng dì-ø-fu-ná

la pauvreté di-ACC-toi-ACT

La pauvreté a mangé toi (tu as un manque cruel d'argent)

(54) b A kwon-náng di-ø-tí-ná

l'eau-pauvreté di- ACC-nous- ACT

La pauvreté de l'eau a mangé nous (nous avons une pénurie d'eau)

104 La différence par rapport au cas précédent, c'est qu'ici la relation S - O est réversible, comme nous l'avons vu avec l'usage processif «brûler», mais cette fois en excluant l'aspect inaccompli :

(54) c Téngnule dènd dí-ø-ń ká-nang

Tengnule propriétaire di-ACC-ACT mil-pauvreté

Les gens de Tengnulé manquent de mil (les gens de Tengnule sont en disette)

Toutes ces tournures insistent sur l'aspect qualitatif de l'événement. Par contre pour dire que la sécheresse sévit partout (dans la durée et dans l'espace), on préfèrera le verbe ny $@ g$ « attraper », éventuellement à l'inaccompli 
(54) d a wád nyəg-ø-ná téng

la sécheresse attraper-ACC-ACT pays

La sécheresse a attrapé le pays (la sécheresse sévit dans le pays).

(54) f a téng nyog-de-ná wád

le pays attraper-INACC-ACT sécheresse

Le pays attrape la sécheresse (la sécheresse sévit dans le pays)

\section{3 - sujet pronom + objet inanimé : le sarcasme}

Ici, nous ne sommes pas loin de la donation du nom, dans la mesure où le schème prédicatif est «S di O n est un simple pronom qui réfère à l'objet :

(55) a a di-ø-ná sínbie nı yúod * a di-de-ná

Elles di-ACC-ACT arachides avec nom

Elles ne portent que le nom d'arachides (ça, des arachides? non)

Ceci veut dire que l'appellation $O$ donnée à $S$ par un interlocuteur est contestée par l'énonciateur, soit parce que la quantité est insuffisante, soit par ce que la qualité est insuffisante. Lorsqu'il s'agit, comme ci-dessus, de désigner des produits alimentaires en eux-mêmes tels que des arachides, du mil, du maïs, des œufs et la viande, le locuteur porte un jugement dépréciatif sur la quantité : "le produit que tu m'as donné est si petit que sa valeur se réduit à sa dénomination". Autre exemple :

(55) b a nénd fuon kù má yúod-ú k-u di

la viande toi donner moi nom c'est qu'elle di-

La viande que tu m'as donnée, ce n'est que le nom qu'elle porte

Par contre, lorsqu'il s'agit d'un aliment qui a été apprêté comme par exemple la bière du mil, la sauce, etc., ce qui est visé, c'est la qualité du processus de production et, par voie de conséquence, celle du produit résultant :

(55) c a di-ø-ná dáan ní yúod * a did-de-ná

elle di-ACC-ACT bière du mil avec nom * inacc

Elle porte le nom de bière de mil ("ça, du dolo, non, c'est du pipi de chat ! »).

Il arrive que l'expansion «n dépréciatif est toujours porté sur la quantité :

(55) d a di-ø-ń sínbie * a did-de-na

elles di- ACC-ACT arachides * inacc

Elles s'appellent arachides (la quantité est si réduite qu'elles ne portent que le nom d'arachides)

(55) e nénd n-ú k-u dì * dìd-de

viande c'est que-elle di- * inacc

C'est de la viande qu'elle s'appelle (elle est si réduite en quantité qu'elle ne porte que le nom de viande)

Dans le dernier exemple, l'antéposition de l'objet est due à la focalisation, le sujet étant représenté par le pronom anaphorique «㺝》. Le statut obligatoirement pronominal du sujet indique que ce genre de contestation ne peut intervenir que dans un contexte où l'existence de l'objet (la viande, les arachides) est pré-construite.

\section{4 - sujet pronom + objet animé}

En fait, ce pronom sujet indique que ce terme est vide. De ce fait, cette tournure garde son sens péjoratif même avec un être animé, puisque celui-ci figure en position objet. S'il 
s'agit d'un animal, le sarcasme peut porter sur la quantité, par exemple si le client pense à la viande que l'animal représente :

(56) a núon n-v́ k-v dì

poule c'est que-elle di

Elle s'appelle poule (mais par sa taille, elle n'est poule que de nom)

112 Mais prononcé par celui qui élève la bête, ce genre d'énoncé peut exprimer l'apitoiement sur l'état de l'animal qui, s'il n'a plus que la peau sur les os, n'est pas loin de mourir.

(56) b u di-ø-ń bú

elle di-ACC-ACT chèvre

Elle s'appelle chèvre (mais elle est si petite qu'elle n'est chèvre que de nom)

113 Lorsque l'objet est un humain, le jugement porte toujours sur la qualité et jamais sur la quantité. Ici, l'évacuation de la position sujet a une portée tragique : les énoncés (56a) et (56b) sont proférés quand une personne entre dans l'agonie, ou donne des signes visibles d'affaiblissement grave; objectivement vivante, certes, mais dans notre esprit, déjà morte :

(57) a v di-ø-ná nlíd * v did-de-na

il di- ACC-ACT il di- ACC-ACT

Il s'appelle personne (il n'est plus une personne que de nom)

(57) b níd-ú k-v dì * dìd-de

personne-c'est que-elle di-AOR

Elle n'est une personne que de nom, mais elle n'en est plus une.

\section{6 - Problèmes d'interprétation}

114 L'extrême diversité des acceptions du verbe di pose de nombreux problèmes d'interprétation. Avant toute chose, je souligne que le verbe di ici étudié garde dans tous les cas les mêmes propriétés morpho-phonologiques, notamment avec le même ton bas et les variations tonales régulières en dagara. Mais les propriétés sémio-syntaxiques font problème : on est en droit de se demander s'il s'agit du même verbe dans les usages processifs et dans les usages statifs. Récapitulons :

\begin{tabular}{|c|c|c|c|c|c|}
\hline $\begin{array}{l}\text { processif } \\
\text { Sujet animé } \\
\text { nutrition } \\
\text { réparation } \\
\text { haut degré } \\
\text { Sujet inanimé } \\
\text { détérioration }\end{array}$ & $\begin{array}{l}\text { exemples } \\
\text { mange le tô } \\
\text { mange le meutre } \\
\text { mange la branoure } \\
\text { Sbrîle } O, O \text { s'use }\end{array}$ & $\begin{array}{l}\$ \\
2.1 \\
2.3,2.4 .2 .5 \\
2.6\end{array}$ & $\begin{array}{l}\text { statif } \\
\text { Sujet animé } \\
\text { dénomination } \\
\text { succès } \\
\text { ressemblance } \\
\text { Styjet inamimé } \\
\text { souffrance } \\
\text { pénurie } \\
\text { sarcasme }\end{array}$ & $\begin{array}{l}\text { exemples } \\
\text { s'appelle Pénu } \\
\text { mange la parole } \\
\text { ressemble à Pénu } \\
\text { anus me mange } \\
\text { paurveté me mange } \\
\text { manger O de nom }\end{array}$ & $\begin{array}{l}5.1 \\
5.2 \\
5.3,5.4\end{array}$ \\
\hline
\end{tabular}

\section{1 - Eventualité de l'homonymie}

115 On pourrait émettre l'hypothèse d'une homonymie possible qui résulterait de l'évolution phonétique de plusieurs étymons du proto-gur: *di // *de, *du, *ti, *ni, *si ... Cette hypothèse est à écarter pour plusieurs raisons. D'abord, nous n'avons trouvé, dans les langues de la famille Gur, aucun signifiant de forme [dentale + voyelle haute antérieure ] qui traduirait une acception de di différente de «manger ». Alors que $80 \%$ des langues de ce groupe ${ }^{14}$ traduisent «manger » par une forme « di, ri, de », et $20 \%$ par une forme « yi, ye ». En remontant plus loin, dans la grande famille Niger-Congo, il existe certes des 
étymons [dentale + voyelle haute antérieure] qui expriment diverses nuances de " être " et " avoir $~^{15}$ et qui pourraient se manifester en dagara actuel dans les acceptions statives de $d i$ (être $\mathrm{x}$ au plus haut degré ou avoir la jouissance de $\mathrm{x}$ ) : en Igbo, être $=$ « di » (be describable as -) vs « bu » (be identifiable as -); être quelque chose = «ni » en Yoruba et en Swhali, être quelque part = « ri » en Shona, etc. Mais le fait que l'étymon *Dv veut dire manger dans un nombre considérable de langues Niger-Congo suggère justement que l'affinité «manger - être " pourrait remonter à la nuit des temps. De toute façon, je

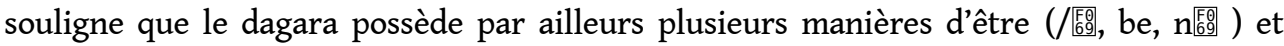
plusieurs manières d'avoir ( $\mathrm{t}_{\mathrm{E}}^{\mathrm{E}} \mathrm{d}$, so), ce qui affaiblit sérieusement la probabilité d'une étymologie *di = avoir ou être.

Par ailleurs, dans d'autres langues oti-volta, le verbe "manger " présente un éventail d'acceptions également large. En mooré, on relève pour di les acceptions : manger, dépenser, gaspiller, consumer, envahir, profiter de, user du pouvoir (régner), assumer la responsabilité d'un acte, être initié, recourir au sortilège, être tranchant. Certes, les deux langues mooré et dagara se ressemblent beaucoup; mais contrairement au dagara, le mooré a une morpho-phonologie très conservatrice, ce qui rend l'homophonie encore moins probable en mooré qu'en dagara. De plus, la séparation ethnique remonte au douzième siècle et, du point de vue culturel, les deux peuples diffèrent à tous égards. Enfin, si l'on se tourne vers le gulimancema, langue nettement plus distante à l'intérieur du groupe oti-volta, on trouve pour di les acceptions : manger, tuer, être nommé chef... dans un simple lexique bilingue ${ }^{16}:$ on en trouverait d'autres dans un dictionnaire plus approfondi.

117 A ce stade de la réflexion, l'hypothèse de l'homophonie est donc simplement improbable. Pour exclure cette hypothèse, il me paraît raisonnable de penser que la démonstration de l'unité sémantique de di est une condition nécessaire et suffisante. Je vais ici tenter de relever ce défi, fort du constat que les usages statifs et processifs de di ont au moins un point commun, à savoir l'attribution d'un nom abstrait au sujet, traduisant l'expression d'un jugement sur lui (l'expression du haut degré et celle du succès).

\section{2 - Approche cognitiviste vs constructiviste}

118 A cette fin, on pourrait supposer que toutes les acceptions de di seraient dérivées d'une signification de base. Mais le concept « sens de base » soulève des interrogations : s'agit-il du sens le plus fréquent? ou du premier sens qui vient à l'esprit? Et de quel point de vue : du point de vue psycholinguistique ou de l'apprentissage? ou du point de vue descriptif et théorique?

119 Selon les linguistes "cognitivistes ${ }^{17}$, le sens est basé sur l'expérience du corps et sa diversification est élaborée à travers des figures comme la métaphore et la métonymie. Une catégorie sémantique donnée est organisée autour d'un "prototype », c'est-à-dire le membre le plus « saillant » de cette catégorie et avec lequel tous les autres membres sont en relation. Dans le cas qui nous intéresse, si l'on demande à un Dagara ce que le mot di évoque pour lui, il mentionne d'emblée la pâte de mil. Et le plus souvent, il porte la main vers la bouche ouverte, doigts assemblés. Le prototype de ce verbe polysémique est donc effectivement "manger». Pour le verbe «/ $\mathrm{w}_{\mathrm{g}}^{\mathrm{F}} \mathrm{b}$ ", croquer, le locuteur interrogé mentionne la viande ou les arachides. Et cette fois, le geste est différent : mouvement des mâchoires, bouche fermée. L'histoire de l'humanité permet d'imaginer que le signe «/ W[5 
des céréales et la maîtrise du feu. Les connotations axiologiques de di montrent également que ce concept a été élaboré au sein d'une société organisée et dotée de normes.

Cependant, on remarquera que le prototype est, par essence, une mise en relation: manger -tô, aboyer-chien, chaise-homme qui s'assied. Par conséquent, plus le concept est abstrait, plus le concept de "saillance" devient problématique et moins la relation

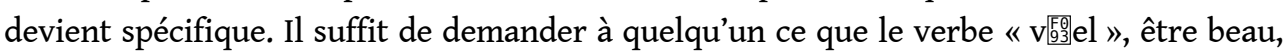
évoque pour lui : il mentionnera une personne générique ou énumèrera des objets quelconques en disant qu'il aime les regarder. Or le propre d'une relation est d'être construite ; dans cette perspective, le prototype apparait, non comme une donnée, mais comme un effet dont la source doit se situer à un niveau plus abstrait ${ }^{18}$.

Même en admettant qu'un mot possède un sens prototypique (vs des sens dérivés), celuici n'est qu'un contenu privilégié parmi l'ensemble des contenus pouvant être construits à partir de la même "forme" (Gestalt). Ce qui a engendré le mot "jambe", c'est une configuration mentale, telle que ce concept distingue l'avant de l'arrière ou non, et telle que ce concept - dès le départ - puisse ou non être appliqué aux animaux, aux arbres et même au soleil : étant donné que pour le Dagara le soleil se déplace, ses rayons sont des jambes. Cette "forme" n'est donc ni concrète ni statique: elle peut impliquer un mouvement, une visée et une temporalité.

Enfin, en admettant que la «saillance » est un phénomène contrôlable chez l'adulte (ce qui n'est pas toujours le cas), il n'en va pas de même chez l'enfant. Celui-ci n'accède pas directement au prototype : ce n'est que progressivement qu'il organise mentalement le contenu des signes. Je souligne que le "mot » di concerne le locuteur dès la toute petite enfance (je ne parle pas de "l'acte " de manger, bien-sûr). Or l'acception "manger " cohabite manifestement, dès cet âge-là, avec l'acception "s'appeler». Ces deux acceptions «manger» et "s'appeler» sont d'ailleurs introduites et utilisées officiellement durant la cérémonie de la donation du nom à l'enfant. Durant cette cérémonie, l'enfant « mange » un nom, et « mange » du beurre en symbole d'accès à la vie des humains. Or l'appartenance contemporaine de ces deux usages de di au vocabulaire de l'enfant est intéressante à plusieurs égards, car dès la petite enfance, la compétence du locuteur inclut des schèmes de fonctionnement sémantique reposant sur des catégories paradigmatiques (action / état; stable, instable) et syntagmatiques (objet friable / non friable ; concret / abstrait). Et par rapport à « vomir », on peut même imaginer la prise de conscience de la catégorie Intérieur / Extérieur et corollairement Bon / Mauvais.

Enfin, l'idée selon laquelle le sens serait basé sur l'expérience du corps est une " évidence ", et en tant que telle trompeuse. En dagara, on ne comprendrait alors pas pourquoi l'épaule est une " calebasse », le mollet un « nuage ", l'œil un " grain », etc. Mais l'essentiel n'est pas là : que le corps soit premier ou non, il nous faut en tout cas envisager par quelles opérations le locuteur parvient à désinvestir et réinvestir la forme sémantique pour engendrer les différentes acceptions du mot. On peut représenter ces opérations à l'aide d'un exemple classique : 


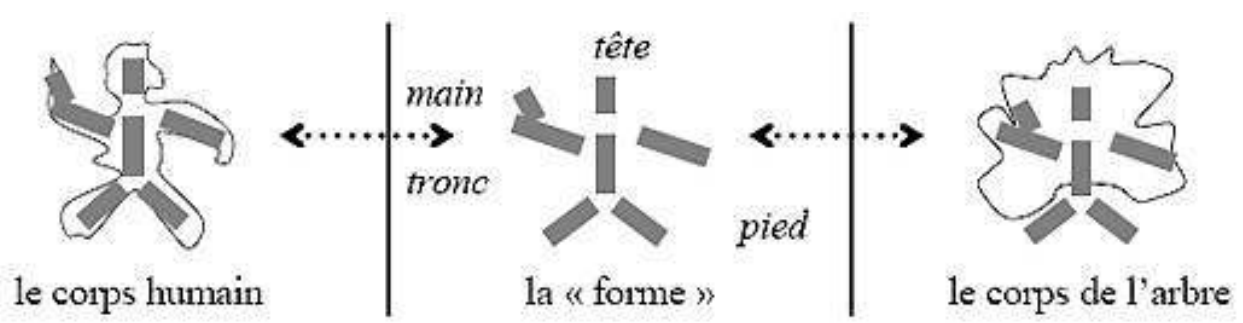

C'est à partir de là que les tenants de la Théorie des Opérations Enonciatives envisagent d'aborder les problèmes de polysémie: « $L a$ tâche du sémanticien est un travail de désintrication visant à caractériser les unités en termes de configurations de paramètres opératoires, en deçà des investissements sémantiques à travers lesquels on les appréhende en première observation. Ces paramètres sont constitutifs d'opérations (ni conscientes, ni perceptibles) qui s'engendrent en interaction avec l'environnement textuel. $»^{19}$

\section{7 - Le verbe DI : invariant et variations}

L'ensemble des acceptions du verbe di peut être dérivé d'une caractérisation générale appelée «invariant ». Celui ne correspond pas directement à aucune des significations citées, mais permet de les embrasser toutes, y compris celles qui semblent contradictoires. Il va de soi qu'on ne pourra rendre compte de cet invariant qu'à un niveau abstrait qui justifie un certain degré de schématisation que, par égard pour le lecteur, je m'efforcerai de minimiser.

\section{1 - le format notionnel de DI}

D'abord, la dualité processus / état est une propriété qui découle du statut de di en tant que verbe d'accomplissement, contrairement à $/ w_{\text {F⿱ }}^{\mathbb{F}} b$ qui est un verbe d'activité pure. Un accomplissement consiste en effet à passer d'un état stable 1 à un état stable 2 au terme d'un processus ayant une certaine durée : l'accomplissement débouche sur un résultat : si j'ai cassé la branche (processus), alors la branche est cassée (état), elle n'a plus à être cassée. Tandis que l'activité pure ne se définit que dans la durée car, au terme du processus, on aboutit à un "vide" : si j'ai lu un livre, le livre n'a pas changé d'état ; mais moi, je ne lis plus, je ne peux que me remettre à lire.

La classification "états, activités, accomplissements" correspond respectivement à la distinction "compact, dense, discret" empruntée à la topologie mathématique et appliquée aux langues naturelles dans la théorie culiolienne ${ }^{20}$. Dans cette perspective, l'énoncé construit autour d'une notion discrète comme di est représentable comme un domaine structuré par une Frontière correspondant à la classe des instants qui permet de passer d'un Extérieur (E) vers un Intérieur (I). Alors qu'une notion dense comme "/

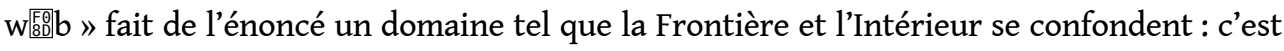
une activité qui n'est validable que dans le processus et qui, à son terme, débouche sur un Extérieur. 

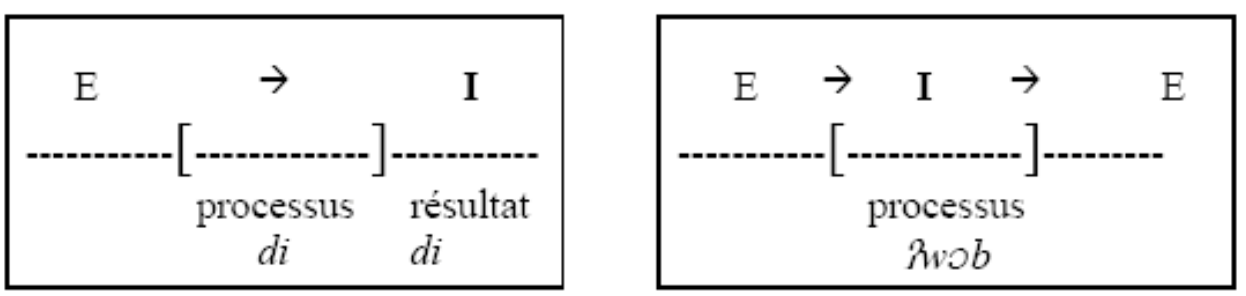

Quant aux états, ce sont des notions compactes, qui caractérisent un domaine notionnel dont la frontière est vide: l'Intérieur et l'Extérieur sont strictement séparés, sans classe des instants qui permette de passer de l'un vers l'autre :

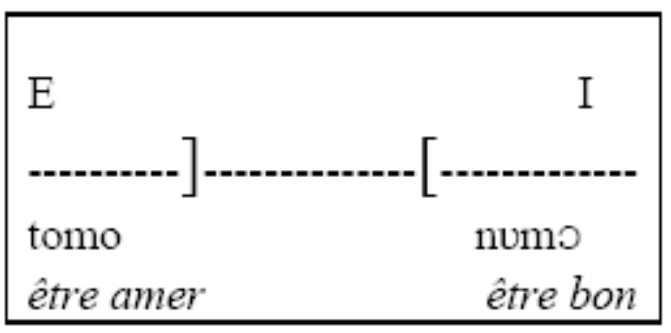

\section{2 - Rôle des paramètres de l'énonciation dans la sémantique de DI}

En fait, le format discret, dense ou compact des notions est déterminé par rapport au point de vue de l'énonciateur qui est considéré, dans la Théorie des Opérations Enonciatives, comme le repère-origine de l'énoncé, et noté Sit $(\mathbf{S}, \mathbf{T})$. Un mot prononcé dans le discours effectif est forcément déterminé par ce repérage subjectif qui, dans l'appréhension de l'événement, pondère le point de vue spatio-temporel («quantitatif » Qnt) et le point de vue qualitatif (Qlt). Les deux types d'usage de di (processif et statif) correspondent à une pondération différente : Qnt \& Qlt vs (Qnt) Qlt. En dagara, tous les verbes discrets sont bivalents car ils sont « compactables » : les verbes d'accomplissement permettent de centrer l'énonciation sur l'état résultant, en faisant abstraction du processus qui y conduit.

Ce formatage explique en partie la valorisation inhérente à di et qui est absente avec / $w$ F⿱ F⿹ $b$, comme nous l'avons vu. Pour le locuteur dagara, si l'argent est "croqué", le résultat est nul ; si l'argent est "mangé", ça sert à quelque chose. De même, di se distingue nettement de l'autre verbe d'absorption, à savoir «nyu ", boire. D'abord parce que ce dernier ne connaît pas d'emplois statifs. Ensuite, parce que «nyu » ne peut avoir de sujet inanimé. Enfin, parce que «nyu » n'implique pas la même valuation que di. Certes, boire l'eau, c'est important pour survivre. Mais, contrairement au to qui est la nourriture de vie et un plat élaboré, l'eau est un élément naturel et ambigu chez les Dagara : on peut dire « 蓲 nyu@-

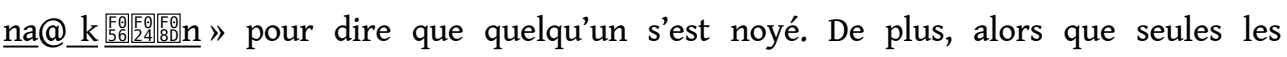
nourritures vitales sont mangeables avec $d i$, n'importe quel liquide est buvable, y compris les choses secondaires, voire mauvaises pour la santé et pour le comportement social, comme l'alcool et le tabac. Fumer se dit : « boire la cigarette».

131 Cependant, le formatage «discret » ne suffit pas à tout expliquer, et ce pour plusieurs raisons. D'abord, le paramètre qualitatif n'intervient pas de la même manière avec tous les verbes discrets. L'action de " casser ", par exemple, peut être bénéfique, maléfique ou indifférente, selon le contexte. Tandis que di n'est pas un verbe d'accomplissement quelconque car dans toutes ses acceptions, la validation fait intervenir une valorisation 
orientée en fonction d'une norme, qu'il s'agisse de la santé, ou de l'intégration dans la société, de réparation ou au contraire de détérioration, et qu'il s'agisse de louange, de critique ou de sarcasme. On a donc besoin de distinguer la "validation » du procès (affirmation / négation) et sa " valorisation » (bon /mauvais). De plus, la référence à une norme ne fait sens que dans la relation entre le sujet parlant et sa communauté, dont l'interlocuteur n'est qu'un représentant. C'est pourquoi la sémantique touche forcément à l'ethnologie.

\section{3 - Nature et Fonction dans la sémantique de DI}

Si l'on veut décrire avec précision les opérations qui engendrent le sens, il faut tenir compte des deux « couches » présentes dans le contenu sémantique du mot. La tradition rhétorique parle depuis longtemps d'analogie de forme ou de nature et d'analogie de fonction. Pour être bref, ce que A. Delplanque ${ }^{21}$ appelle la " Nature » sémantique d'un mot répond à la question "qu'est-ce que c'est?». Par exemple, la cendre est une poudre grise; piler, c'est frapper avec un pilon. Et ce qu'il appelle la «Fonction» sémantique permet de répondre à la question : «A quoi ça sert? ou d'où ça vient? ». Par exemple, la cendre résulte de la combustion; piler a pour effet de réduire le grain en poudre.

A travers ses diverses acceptions, la Nature de di peut être glosée de la manière suivante: le sujet est le point d'arrivée ou l'attributaire d'un objet, ce qui peut se représenter par la visée

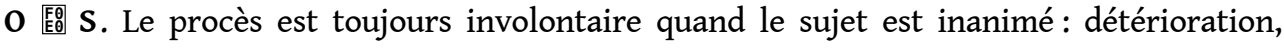
souffrance, pénurie, sarcasme. Le procès ne peut être volontaire que si le sujet est animé : nutrition, réparation et renomination. Mais il peut être involontaire même si le sujet animé.

- Dans les trois cas où di a le sens de «manger ", le sujet s'attribue volontairement l'objet (nutrition, rapports familiaux, réparation);

- C'est l'énonciateur qui attribue la propriété $O$ au sujet $S$ dans les cas suivants : dénomination et renomination, ressemblance, haut degré, succès, sarcasme ;

- Dans le cas de la détérioration, de la souffrance et de la pénurie, l'objet O n'est pas " affecté » par $\mathrm{S}$ (contrairement à ce qui se passe avec les quasi-synonymes : croquer, attraper) mais, dans l'inconscient dagara, englouti par lui, ou si l'on veut, anéanti « en lui ». Je signale qu'en dagara, ce n'est pas l'homme qui a faim ou soif ; c'est la faim et la soif qui ont l'homme.

A travers ses diverses acceptions, la Fonction de di peut être glosée de la manière suivante: le procès effectue un centrage qualitatif tel que, étant donné une propriété $Q$ et son contraire Q', l'une de ces deux valeurs est exclue.

- Lorsque le sujet est inanimé, on obtient toujours une valeur détrimentaire : $\mathbf{Q}$ 嵑 $\mathbf{Q}$. C'est le cas de la détérioration, de la souffrance, de la pénurie et du sarcasme.

- Lorsque le sujet est animé, l'effet est toujours avantageux : Q' 瞖 Q. Cette valorisation correspond aux acceptions suivantes: le haut degré et le succès (exclusion de la valeur médiocre), la nutrition, y compris dans le cas du repas propitiatoire (survie de l'individu et conformité au code social), la ressemblance parfaite (élimination de la différence) et la dénomination. Dans ce dernier cas, il s'agit bel et bien de donner à l'individu une propriété qui le distingue de tous les autres individus : Q par rapport à Q', Q"22. 


\section{4 - Rôle des instances de l'énonciation dans la sémantique de DI} parlé) 47sd), etc.

Ces deux dimensions sémantiques du mot, Nature et Fonction, sont concomittantes dans l'énonciation: elles ne sont ordonnées ni dans le temps ni dans l'espace, et l'une ou l'autre peut être occultée. Et elles sont toutes deux le cadre d'opérations spécifiques de génération de sens : commutations (type métaphore) ou transferts (type métonymique).

Mais ce que A. Delplanque ne dit pas, c'est à quel(s) niveau(x) énonciatif(s) correspondent ces deux ensembles, à quoi ils correspondent dans ce que A. Culioli appelle la " cascade " des opérations énonciatives : Sit2 - Sit1, Sito - Sit'o. Mon hypothèse est que la Nature du mot est appelée à s'actualiser dans l'énonciation : à ce niveau on se préoccupe de savoir qui « mange » quoi et comment, selon que le sujet et l'objet sont \pm animés. Tandis que la Fonction est appelée à s'actualiser dans la co-énonciation : c'est à ce niveau que l'on peut mesurer la conformité de ce qui est validé par rapport à ce que les « autres » attendent, espèrent ou redoutent (par exemple dans l'expression de la réussite : bien joué, bien

Cette hypothèse pourra surprendre le lecteur. D'abord, je souligne que nous travaillons ici sur la contribution du mot au sens de l'énoncé, et non sur la syntaxe du discours, où la relation co-énonciative intervient notamment dans la construction du mode: interrogation, injonction, hypothèse, etc..$^{23}$ - Cela dit, le mot est fait pour être énoncé dans le discours. Disons plus exactement que le mot est un « énonçable » et, à sa manière, contient déjà toute la problématique de l'énonciation. Si le format notionnel du mot (discret ou compact) favorise l'activation de tel ou tel paramètre de l'énonciation $(\mathrm{S}, \mathrm{T})$ ou (Qnt, Qlt), le mot peut tout aussi bien favoriser l'activation de telle et telle instance énonciative (Sit2, Sit1, Sito, Sit'o). Ainsi, le mode est-il souvent solidaire du choix du verbe : par exemple dans le cas de « souhaiter + subjonctif » vs « espérer + indicatif ». Par ailleurs, des verbes comme « répéter » ou « confirmer » impliquent deux actes d'élocution successifs, une situation qui, au niveau du discours, peut provoquer certains effets agréables ou désagréables sur l'auditeur.

Enfin, le sémanticien doit décider si les connotations du mot, les niveaux de langue, les devoirs et les tabous font partie de son champ d'étude, ou si ces phénomènes relèvent de la compétence du stylisticien, du sociolinguiste ou de l'ethnologue. Il me semble qu'A. Culioli a déjà répondu à cette question, en définissant la notion comme un faisceau de propriétés "physico-culturelles ». Dans le cas de di en dagara, je crois donc que la coénonciation est à l'œuvre dans l'effet de provocation signalé à propos du haut degré (ex. 28), dans la volonté de s'affirmer face à aux autres en changeant de nom (ex. 39), dans le caractère plus ou moins péremptoire de l'expression de la ressemblance (ex. 46a - 48), ainsi que dans le tabou signalé à propos de la comparaison avec des parents proches (ex.

En admettant cette hypothèse - ou en négligeant ce qui n'est peut-être qu'un problème terminologique - tout se passe comme si le point de vue adopté par le co-énonciateur dépendait à la fois de la nature \pm animé du sujet et de la qualité escomptée. En notant le sujet animé par S+ et le sujet inanimé par S- et en notant Nature et Fonction par Sito et Sit'o respectivement, cela donne la représentation suivante : 

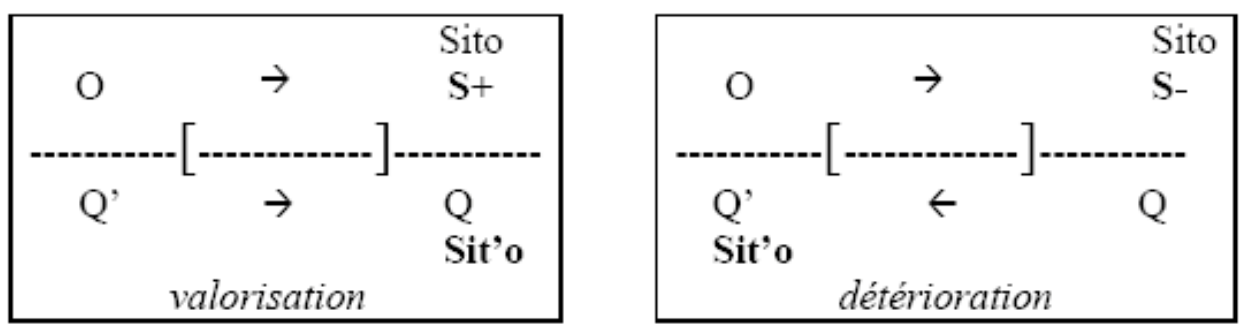

On pourrait réduire ces deux schémas en un seul, en utilisant des variables \pm . L'essentiel est qu'en définitive, on peut gloser ainsi l'invariant de di à travers la diversité de ses acceptions :

L'attribution d'un objet ou d'une propriété au sujet a pour effet de radicaliser la position de celui-ci dans un domaine que l'Enonciateur juge vital pour le sujet lui-même ou pour la société.

Dans ce cadre général, parmi les configurations possibles, le sens prototypique de « manger » n'est que la configuration optimale : sujet animé et volontaire, objet inanimé, effet bénéfique pour le sujet et pour la société.

\section{BIBLIOGRAPHIE}

Alexandre R. P. (1953). La langue möré. Mémoires de l'Institut Français d'Afrique Noire, n 34 , Dakar.

Bendor-Samuel, J. T. (1989). The Niger-Congo languages: a classification and description of Africa's largest language family. ed. Rhonda L. Hartell, Lanham, MD: University Press of America.

Culioli A.(1990-1999). Pour une linguistique de l'énonciation (tomes 1, 2 \& 3), Ophrys, Paris.

Delplanque A.(1986). La langue dagara, essai de sémiologie linguistique. Thèse d'Etat, Université de Paris VII.

Delplanque A. \& al. (2006.) L'expression de l'apparence. Travaux menés au sein de l'équipe «Langues \& Représentations » de l'Université François-Rabelais de Tours en 2004 - 2005, resp. A. Delplanque ; université de Poitiers, in CORELA (revue électronique)

Delplanque A.(à paraître 2007). Forme et Déformabilité, Université de Tours \& Université de Ouagadougou.

De Voguë S. (1989). « Discret, dense, compact : les enjeux énonciatifs d'une typologie lexicale » in : La notion de prédicat, éd. JJ. Franckel, collection URA 1028, Université de Paris VII.

Fauconnier G. (1984). Espaces mentaux, Paris : Editions de Minuit.

Franckel JJ. \& Lebaud D. (1990). Les figures du sujet, à propos des verbes de perceptions, sentiment, connaissance, Paris, Ophrys.

Franckel JJ. \& Paillard D. (1991). « Dense-discret-compact : vers une typologie opératoire » in: Travaux de linguistique et de philologie, xxix, éd. G. Kleiber et G. Roques, Strasbourg-Nancy, Klincksieck. 
Franckel JJ. \& Lebaud D. (1992). "Lexique et opération - Le lit de l'arbitraire", in La théorie de A. Culioli, ouvertures et incidences, Paris. Ophrys, p. 89-105.

Janda L. (Febr. 2000). "Cognitive Linguistics", Sling2K Workshop: Cognitive Linguistics, University of North Carolina.

Kleiber G. (1990). La sémantique du prototype, Presses universitaires de France.

Langacker R. (1987 / 1991). Foundation of Cognitive Grammar, Stanford University Press, vol. 1 / 2.

Lakoff G. (1987). "Women, fire and dangerous things", University of Chicago.

Manessy G. (1975). Les langues Oti-Volta : classification généalogique d'un groupe de langues voltaïques. SELAF, Paris.

Ouoba B.B. (1983.) Lexique gulimancema-français, Commission Nationale des Langues Voltaïques, Ouagadougou.

Somé P.A. (1982). Systématique du signifiant en dagara : variété wúlé, L'Harmattan, Paris.

Somé P.A. (1992). Signifiant et Société, le cas du dagara du Burkina Faso, Paris L'Harmattan.

Somé P.A. (2003). Linguistique générale : éléments de phonologie, de sémantique et de grammaire du Dagara, L'Harmattan, Paris.

Vendler Z. (1967). « Verbs and Time ", in: Linguistics and Philosophy, Ithaca, N.Y. Cornell University Press.

Welmers W.E. (1973). African Language Structures. University of California Press.

\section{NOTES}

1. Il s'agit plus précisément du parler «wulé ». Je tiens à souligner que, malgré la parenté incontestable, ce parler présente des différences phonologiques, grammaticales et sémantiques, à la fois par rapport aux autres parlers dagara du Burkina (lobr, birifor) et par rapport aux différents parlers «dagaare» du Ghana. L'acceptabilité ou la non-acceptabilité des énoncés présentés ci-dessous est valable pour le parler wulé.

2. La voyelle $-v$ de $-d v$ est réalisée [e / $\varepsilon$ ], si la voyelle de la base est i/l, e/ $\varepsilon$, elle est réalisée [o/o] si la voyelle de la base est $u / v, o / o$, ou si la base verbale, quelle que soit la voyelle de la base, est terminée par les consonnes $-\mathrm{g}$ et $-\mathrm{b}$; enfin, $-\mathrm{v}$ de $-\mathrm{dv}$ est réalisée [a] si la voyelle de la base est terminée par la voyelle a. Voir pour plus de détails P.A. SOMÉ, 1996, 2003

3. Cette forme est acceptable au sens de " les enfants ont frappé », mais jamais au sens passif, car " pób » est un verbe d'activité pure. Alors qu’on pourrait avoir : « a bibiit gáal-a » = les enfants on été couchés, car « gaal » est un verbe d'accomplissement.

4. La classification des verbes en états, accomplissements et activités est due à Z. Vendler (1967).

5. Le « to » est un mot d'origine diula et qui désigne le plat de base des Dagara : une pâte de mil, et non d'igname ou de manioc, qui se mange accompagné de sauce.

6. cf. l'enquête de Delplanque (1986).

7. L'autre parler dagara, le lobr, considère plutôt que le riz est pâteux et « se mange » ( $v d l-d \varepsilon$ ní à muné « il est en train de manger du riz ») et non se « croque » comme c'est le cas en wulé

8. Littéralement : « chose -manger-able-pluriel » et « chose-croquer-able-pluriel ».

9. Ici, « croquer » marque la croyance dagara selon laquelle les sorciers mangent l'âme de leur victime.

10. Dans les autres cas d'adoption, le verbe utilisé est pólı « prendre en héritage ». 
11. Exemple : n won-ø-ná a fù Pyદtu /je/ entendre-ACC-ACT/ la/ ta/ parole / «j'ai entendu ta parole » en fait « j'ai compris tes propos »

12. En dagaare (Ghana), ce "ka" n'apparaît que dans ce contexte complétif, alors qu'il n'apparaît pas dans les cas précédents (36a et 36b). Mais je souligne qu'en wulé et aussi en lobr, l'absence de "ka" donne une toute autre signification qui sera présentée en 39a et 39b.

13. Voir l'ouvrage collectif : “Les verbes d'apparence”, revue CORELA 2006.

14. Comptage effectué dans l'index lexical fourni par G. Manessy (1975). Voir aussi BendorSamuel (1989).

15. Voir par exemple Welmers (1973).

16. Celui de B.B. Ouoba (1983). Pour le mooré, voir le dictionnaire de P. Alexandre (1953).

17. Notamment George Lakoff (1987), Ronald Langacker (1987 / 1991). Voir aussi Laura Janda (2000).

18. Dans ce qu'il appelle une version "étendue" de la théorie cognitiviste, G. Kleiber (1990) fait remarquer que le prototype ne s'applique pas au monde réel, mais au monde "perçu" comme tel. Plutôt qu'une image mentale, le prototype serait plutôt un faisceau abstrait de propriétés typiques. Et ce faisceau ne serait qu'un effet de surface qui ne reflèterait qu'une partie de l'activité mentale de l'homme.

19. Franckel JJ. \& Lebaud D. (1992), p. 89-105.

20. La Théorie des Opérations Prédicatives et Enonciatives. Voir : A. Culioli (1990-1999), S. de Voguë (1989), JJ.Franckel \& D.Paillard (1991).

21. « La langue dagara : essai de sémiologie linguistique » (1986).

22. Rappelons que le nom individuel chez les Dagara n'appartient pas à une liste close, mais constitue un énoncé condensé qui stigmatise l'intention du donneur de nom dans les circonstances de la naissance. Par exemple, le prénom "saan 1 bon" = qu'est-ce qu'un père?, donné au dernier-né après une querelle entre le père et le fils aîné.

23. Voir A. Culioli (1990-1999), tome 1.

\section{RÉSUMÉS}

En dagara, la première traduction qui vient à l'esprit pour le verbe di est «manger. De là, on obtient l'idée de « dépenser, profiter » et, de manière moins évidente, celle de «brûler, être usé, faire souffrir, être infecté ... ", mais aussi, de manière paradoxale, "s'appeler, avoir les mêmes propriétés que $\mathrm{x}$ », puis « n'être $\mathrm{x}$ que de nom » ou au contraire « atteindre l'excellence, atteindre son but, normaliser une situation "...Dans chacune de ces acceptions, di exprime toujours une valeur marquée par rapport à ses faux-synonymes (croquer, flamber, se lustrer, faire mal... appeler, être ou avoir, ressembler, réussir, réparer, etc). On distingue deux types d'emplois, l'un comme verbe d'accomplissement, l'autre comme verbe d'état. Dans les deux cas, les acceptions sont classées en fonction de la nature $\mathrm{du}$ sujet et $\mathrm{du}$ complément. L'analyse révèle progressivement comment la structure grammaticale engendre le sens, en conformité avec les données ethnologiques, et décèle une valuation constante de l'état des choses par l'Enonciateur. Après une brève discussion sur l'approche cognitiviste vs constructiviste, l'article tente de dégager l'invariant qui fait l'unité de toutes les acceptions de di.

In Dagara, the most common translation for the verb di is « eat ». Other translations, however, are: "spending, taking advantage of" and, more surprisingly, that of "burning, wearing out, 
hurting, being infected...", but also "be named x, look like x", then "be x only by name" or, on the contrary, "be topmost, reach the target, make good for a bad situation". For each of these meanings, "di" always differs from its false-synonyms (munch, blaze, wear, hurt... call, be or have, resemble, manage, make up for...). We distinguish two main types, one where di is a verb of accomplishment, and one where di is a verb of state. In both cases, the meanings are classified according to the nature of the subject and of the complement. The investigation gradually reveals how the grammatical structure fits with the semantics as well as the ethnological data, mainly through a constant valuation of the state of affairs by the Speaker. After a brief discussion on the cognitivist vs constructivist approaches, the article concludes by showing how all of the meanings can be united around a single common, abstract schema.

INDEX

Mots-clés : langues gur, polysémie, énonciation, constructivisme, invariant, aspectualité, sujet animé-inanimé, objet concret-abstrait

Keywords : Gur Languages, polysemy, enunciation, constructivism, aspectuality, concrete / abstract object

\section{AUTEUR}

\section{PÉNOU-ACHILLE SOMÉ}

École Pratique des Hautes Études 